\title{
IDEAS DE LOS ALUMNOS SOBRE LA DIGESTIÓN: ASPECTOS FISIOLÓGICOS
}

\author{
BANET, E. y NÜÑEZ, F. \\ Escuela Universitaria de Magisterio. Murcia. \\ C.P. San Isidoro. Murcia.
}

\section{SUMMARY}

In this article we report the ideas owned by students from different leveis (EGB, BUP, Teacher Training College) as well as by some teachers, in relation to certain concepts involved in the processes of digestion and substance absorption.

The dificulties observed in the conceptualization of several processes, especially in lower lever students, show the need for a change in the teaching planning.

\section{INTRODUCCIÓN}

El estudio de los conocimientos previos de los alumnos ha constituido, en los últimos años, un punto de referencia importante en la investigación en Didáctica de las Ciencias. Se ha obtenido, así, información sobre importantes errores que tienen los estudiantes de distintos niveles educativos, en diferentes áreas del conocimiento. Se trata en muchos casos, como ponen de manifiesto diversos especialistas en la materia (Giordan 1982, Coll 1985, Driver 1986, Gil 1986), de esquemas conceptuales alternativos al conocimiento científico, muy arraigados en la estructura cognitiva del alumno y con un nivel muy importante de coherencia interna. Por todo ello, estos errores son difícilmente modificables y pueden interferir negativamente con la nueva información que se intenta transmitir.

Es evidente que el interés de estas investigaciones no radica, exclusivamente, en establecer un catálogo detallado de los errores sobre cada conjunto de lecciones. Aunque el profesor determine los conocimientos previos de sus alumnos y los tenga en cuenta en la planificación de sus actividades de clase, ello no siempre garantiza la progresión conceptual de los alumnos. Así, cuando pretendemos modificar estas nociones equivocadas observamos, con mayor frecuencia de la que seria deseable, cómo estos intentos fracasan. Podemos comprobar, incluso, cómo algunos grupos de alumnos retroceden, en lugar de progresar.

Sin embargo, los conocimientos previos de los alumnos proporcionan datos muy significativos a los responsables de la planificación y desarrollo de un trabajo anterior (Banet y Núnez 1988), en el que analizamos las nociones y representaciones de los alumnos de diferentes niveles educativos en relación con aspectos anatómicos del aparato digestivo. Si entonces señalábamos las dificultades de los alumnos para aprender los rasgos anatómicos de este sistema, no cabe duda que estas dificultades se incrementarán considerablemente, de forma muy particular en los niveles básicos, cuando pretendemos que determinados conceptos como la digestión o la absorción intestinal sean comprendidos de forma correcta.

Si bien el objeto del presente trabajo no es otro que presentar los resultados obtenidos sobre los conocimientos previos de los alumnos en relación con el proceso digestivo y la absorción de las sustancias resultantes del mismo, creemos necesario avanzar en el sentido de conocer la incidencia de propuestas didácticas concretas sobre los conocimientos previos de los alumnos. Por ello, considerando algunas premisas expuestas por Driver (1986), en cuanto a la construcción del conocimiento, asi como algunas aplicaciones de las teorías de Ausubel a la enseñanza de las Ciencias (Novak 1982), hemos desarrollado una serie de intervenciones didácticas (algunas puntuales, otras no tanto), relacionadas con el aprendizaje de conceptos de nutrición humana, cuyos resultados estamos analizando en estos momentos.

\section{POBLACIÓN ESCOLAR. MÉTODOS DE TRABAJO}

Aunque nuestro interés se centra fundamentalmente en el Ciclo Superior de EGB, hemos analizado también Ios resultados obtenidos en $3^{\circ}$ de BUP y en la Escuela Universitaria de Magisterio ( $3^{\circ}$ de la especialidad de 
Tabla I

Población explorada.

\begin{tabular}{|c|c|c|c|c|}
\hline NIVEL & cusso & ALUMNOS & CENTROS & CONDICIONES \\
\hline EGB & $6 ?$ & 159 & 4 & Pre-test \\
\hline EGB & $8 \underline{9}$ & 101 & 3 & Postest \\
\hline Bup * & 30 & 72 & 1 & Pre-test \\
\hline Magisterno & $3 ?$ & 54 & 1 & Pre-test \\
\hline Profesorks" & - & 26 & - & - \\
\hline
\end{tabular}

- Alumnos de la especialidad de ciencias.

- Licenciados en Quínicas, Geología, Matemáticas,

o maestros del área de ciencias.

ciencias), asi como los obtenidos con una muestra reducida de profesores, con objeto de disponer de algunos datos que nos permitan dar un mayor alcance a nuestras conclusiones. En la Tabla I presentamos los detalles relativos a la población estudiada.

\section{CUESTIONARIOS Y ENTREVISTAS}

Para aproximarnos al conocimiento que poseen los alumnos en relación con la digestión de los alimentos y la absorción de las sustancias nutritivas que forman parte de los mismos, hemos centrado nuestra exploración en los siguientes aspectos:

- Determinar cuál es Ia importancia que conceden a los diferentes órganos del tubo, según la intensidad de las acciones digestivas que en cada uno de ellos tiene lugar.

- Nociones que poseen sobre el proceso digestivo, en cuanto a las acciones que implica y a las sustancias resultantes de la transformación de los alimentos $\mathrm{y}$, en consecuencia, conocer cuál es el papel que atribuyen a las diferentes secreciones digestivas.

- Poner de manifiesto las ideas de los alumnos en relación con diferentes aspectos de la absorción de sustancias.

Para ello, hemos combinado la utilización de dos técnicas de trabajo complementarias: los cuestionarios y las entrevistas individuales.

\section{Cuestionarios}

Los cuestionarios han sido elaborados a partir de una entrevista previa a un número reducido de alumnos y reformulados después de haber sido ensayados en aulas experimentales. En su preparación hemos tenido en cuenta las recomendaciones que señalábamos en un trabajo anterior (Banet y Núñez 1988).
A) La primera pregunta del cuestionario nos proporciona amplia información sobre diferentes aspectos del aparato digestivo y la digestión:

«Sobre la siguiente silueta humana, dibuja el camino que recorre un trozo de pan y un vaso de agua cuando penetran por la boca, indicando el nombre de cada una de las partes por las que pasan. Explica el camino que has dibujado".

B) Para intentar conocer lo que piensan los alumnos en relación con el proceso digestivo, hemos planteado las siguientes preguntas:

- Califica de cero a tres los siguientes órganos del tubo digestivo, según la importancia de las acciones digestivas que en ellos tienen lugar:
Intestino grueso Ano
$0=$ Ninguna importancia
Boca Intestino delgado
$I$ = Un poco importante
Faringe Esofago
2 = Bastante importante
Estómago
3 = Muy importante

Señala, mediante una cruz, la frase que mejor explica en qué consiste la digestión:

a) Obtener la energía necesaria para el mantenimiento del organismo; b) transportar los alimentos por el cuerpo; c) descomponer los alimentos en sustancias nutritivas más sencillas; d) separar de los alimentos las sustancias buenas y malas; e) realizar las funciones vitales; f) mezciar el alimento con los jugos para facilitar la digestión; g) absorción de los alimentos.

Explica brevemente la frase que has señalado.

- ¿Qué funciones realizan los jugos digestivos? Escribe los nombres de todos los que recuerdes.

C) Por último, con objeto de analizar las ideas de los alumnos sobre la absorción de las sustancias resultantes de la digestion, hemos incluido las siguientes cuestiones:

- ¿En qué consiste la absorción de sustancias que tiene lugar en el tubo digestivo?

- ¿En qué parte del tubo digestivo se produce fundamentalmente la absorción?

- ¿Qué ocurre con las sustancias absorbidas?:

¿A dónde se dirigen? ¿Cómo se transportan? ¿Para qué son utilizadas?

\section{Entrevistas individuales}

Los dibujos que realizan los alumnos, así como sus explicaciones por escrito, particularmente cuando son consecuencia de preguntas relativamente abiertas sobre procesos complejos, como son los que nos ocupan, ponen de manifiesto contradicciones, en algunos casos bastante importantes. Para obtener las aclaraciones pertinentes y para profundizar más en el conocimiento de sus esquemas conceptuales, en relación con 
estos procesos, es necesario recurrir a Ias entrevistas individuales que, en nuestro caso, han sido realizadas con una reducida muestra de alumnos de EGB y Magisterio, seleccionados de acuerdo con ciertas categorías de respuestas obtenidas a partir del cuestionario. Las preguntas se han centrado fundamentalmente en los siguientes aspectos:

- Importancia del estómago en el proceso digestivo.

- Digestión: En qué consiste y cómo se realiza.

- Absorción: Parte del tubo digestivo donde tiene lugar. Naturaleza de las sustancias absorbidas.

\section{RESULTADOS Y DISCUSIÓN}

\section{Localización de las acciones digestivas}

En la Tabla II presentamos los resultados obtenidos en relación con la valoración de los alumnos sobre la intersidad de las acciones digestivas en diferentes órganos del tubo digestivo. El estómago es considerado el órgano de mayor importancia en todos los niveles estudiados. Las puntuaciones medias que le otorgan los alumnos son muy elevadas, sensiblemente superiores a las del intestino delgado, segundo órgano en importancia.

Tabia II

Intensidad de las acciones digestivas en diferentes órganos del tubo digestivo (\%).

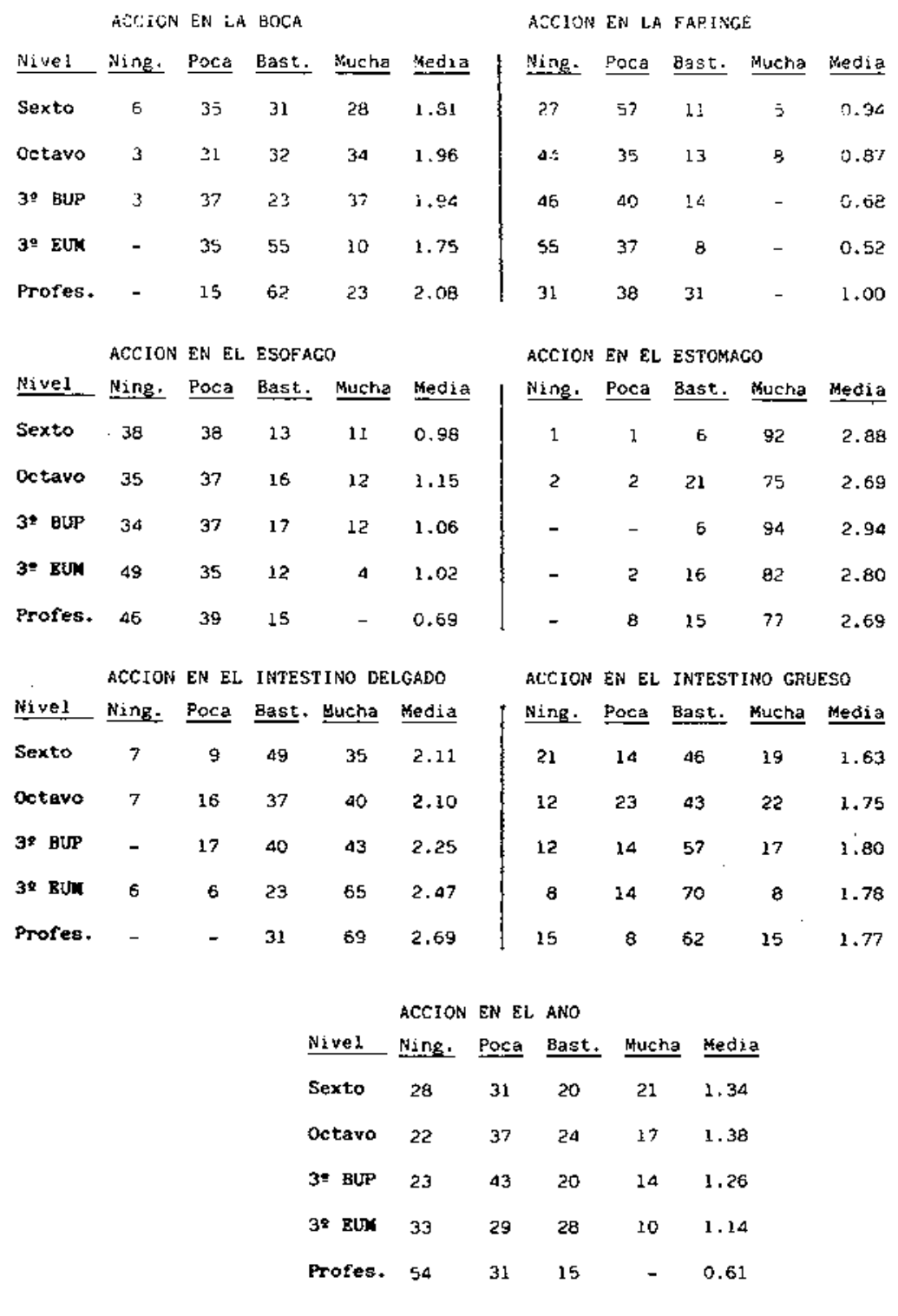


A pesar de las diferencias de instrucción de los alumnos, existe una coincidencia generalizada en lo que se refiere a la importancia relativa que conceden a cada uno de los restantes órganos. Así, después del intestino delgado los sitúan, por este orden: la boca, intestino grueso, ano, esófago y, en último lugar, la faringe.

Conviene setalar, sin embargo, que la importancia que se atribuye a la boca se relaciona, fundamentalmente, con acciones de naturaleza mecánica, ya que, en la mayor parte de los casos, la saliva no se considera como una secreción con acciones digestivas, como veremos después. Por otra parte, las puntuaciones, relativamente elevadas, que se atribuyen al ano (entre el $35 \%$ y $40 \%$ lo consideran bastante o muy importante) se relacionan con la eliminación de heces.

Excepto en Magisterio, donde se reconocen, generalmente, acciones digestivas al intestino delgado, más de la mitad de los alumnos encuestados opinan que la intensidad del proceso digestivo es mayor en el estómago que en el intestino delgado. Esta noción, confirmada mediante entrevistas individuales, constituye en muchos casos un esquema conceptual (no un error puntual) coherente: «El estómago es el órgano central de la digestión». Así, por ejemplo, cabe señalar que aqueIlos alumnos, particularmente entre los de EGB, que conceden la máxima puntuación a este órgano, consideran que el hígado y/o el páncreas vierten sus secreciones en el estómago (Banet y Núñez 1988). En consecuencia, el papel del intestino es explicado por una de estas posibilidades:

- Como receptor de sustancias de desecho, ya que la digestión finalizaria en el estómago, donde tendria lugar la absorción de las sustancias resultantes.

- Como lugar de absorción de los compuestos resultantes de la digestión, pero sin actividad digestiva, ya que el proceso finalizaría en el estómago.

\section{Ideas de los alumnos sobre la digestión}

Existe, pues, una tendencia bastante generalizada a considerar el estómago como órgano central del proceso digestivo. Intentaremos, ahora, proporcionar algunos datos que nos puedan aproximar a sus ideas sobre dicho proceso. En este sentido, presentamos en la Tabla III las respuestas de los alumnos en relacion con la frase que, en su opinion, explica mejor la digestión.

De las diferentes posibilidades ofrecidas, una gran mayoria opta por: «Descomponer el alimento en sustancias nutritivas más sencillas». Sin embargo, cuando se les piden aclaraciones sobre el significado de dicha descomposición, apreciamos la escasa diferenciación del concepto de digestión en la estructura cognitiva del estudiante. Buena prueba de ello son las siguientes explicaciones, muy extendidas entre los alumnos de octavo:

- Los alimentos (que en muchas ocasiones son identificados con las sustancias nutritivas que contienen) están formados por una mezcla de sustancias buenas o aprovechables y por sustancias malas o no aprovechables. Cuando emplean el término "sustancias nutritivas», algunos de ellos no saben indicar ninguna en un alimento concreto, por ejemplo, la leche.

- El alimento, tal como lo comemos, no puede llegar a las células, sino que es necesario descomponerlo. Para ello, se mezcla con los jugos digestivos (a veces una clase de ácidos), que ayudan a la digestión.

- La digestión consiste, además de machacar y triturar los alimentos, en separar las sustancias buenas de las malas contenidas en los alimentos. No es posible obtener mayor información sobre la naturaleza de esta descomposicion o separación.

Tabla III

Concepto de digestion (\%)

\begin{tabular}{|c|c|c|c|c|c|}
\hline Categorias de respuestas & 61 EGB & 8음 & 30 BUP & 3 EUth & Profesores \\
\hline Obtención de energía & 6 & 14 & 5 & 19 & - \\
\hline Iransporte de alimentos & 3 & 5 & - & - & 8 \\
\hline \multicolumn{6}{|l|}{ Descomposición de alimentos } \\
\hline en sistaricias nutritivas & 78 & 67 & 81 & 75 & 84 \\
\hline \multicolumn{6}{|l|}{ Separar sustancias buenas de } \\
\hline las sustancias malas & 5 & 9 & 5 & - & 8 \\
\hline Realizar las funciones vitales & 3 & 2 & 3 & 3 & - \\
\hline Pezclar el alimento con jugos & & & & & \\
\hline digestivos & 4 & 2 & 3 & - & - \\
\hline Absorción de aidmentos & - & 1 & - & 3 & - \\
\hline Otras/NAC & 1 & - & 3 & - & - \\
\hline
\end{tabular}


- Como resultado de la digestión, el alimento se ha descompuesto, partido o triturado en trozos mucho más pequeños (son los mismos alimentos, pero de ta. manto mucho más reducido). Es decir, persiste la idea de una acción exclusivamente física.

En sexto de EGB las ideas que se tienen sobre este proceso son aún más confusas. Existe una amplia varicdad de opciones para definir el proceso digestivo, entre las que seleccionamos las siguientes:

- Algunas demuestran una escasa o nula comprensión del proceso: «Una serie de procesos para asimilar los alimentos para que puedan ser distribuidos por el cterpo»; "Tomar los alimentos y mezclarlos en el estómago con los jugos»".

- Otras pueden considerarse importantes por existir una notable coincidencia entre los alumnos. Asi, uno de cada tres estudiantes entrevistados sentala: «Separar las sustancias buenas y las malas del alimento».

- Algo menos de la mitad de los alumnos utilizan la frase "Descomposición de los alimentos", aunque incorrectamente empleada en muchas ocasiones: «Descomponer los alimentos en jugos gástricos para alimentat al cuerpo».

- Finalmente, señalar que sólo dos, de quince alum. nos entrevistados, dan una explicación de cierta consistencia: «En descomponer los alimentos en sustancias nutritivas más simples», "Las sustancias nutritivas van atravesando el intestino delgado hacia las células y las sustancias de desecho son expulsadas por e! ano»?.

Las explicaciones del proceso digestivo obtenidas en tercer curso de Magisterio son, lógicamente, más preci* sas y elaboradas: "La digestión consiste en una serie de procesos químicos donde los alimentos se descomponen en una serie de sustancias, las cuales el organismo si puede asimilar, como son: proteinas, vitaminas, glúcidos, ...»; "La digestión es un proceso que transforma, mediante una serie de acciones físicas y quími- cas el alimento inicial en sustancias asimilables por ei organismo. En este proceso se separan los componentes de cada alimento de forma que se asimila lo que es aprovechable, el resto se expulsa».

Sin embargo, ello no implica que el grado de comprensión de este proceso sea el adecuado. Al contrario, podemos encontrar, con alguna frecuencia, que estas definiciones, más o menos academicistas, contienen u ocultan imprecisiones de mayor o menor alcance. He aquí unas muestras de ello:

- Aunque se habla de alimentos y sustancias nutritivas, no está suficientemente clara la diferencia entre ambos conceptos. Por ejemplo, en ocasiones se señalan como alimentos la glucosa o las poteínas.

- Cuando se les pregunta por las sustancias nutritivas, se enumeran hidratos de carbono, proteínas, lípidos...; sin embargo, desconocen las implicaciones, a nivel de moléculas concretas, del proceso digestivo. Pongamos un ejemplo, cuando les preguntamos qué compuesto tiene mayor peso molecular, una molécula de glusosa o una proteína, los alumnos suelen dudar en la respuesta, pero al insistir, obtuvimos los siguientes resultados: de 15 alumnos entrevistados, 6 señalan que las proteínas; 5 indican que la molécula de glucosa; 2 manifiestan que según los casos, unas veces scrá mayor la proteina y otras la glucosa; por último, dos no tienen una opinión clara al respecto.

\section{Jugos digestivos}

Después de lo que acabamos de señalar sobre el procc. so digestivo, no resulta extraño que bastantes alumnos, particularmente en los niveles básicos, posean una idea poco precisa en relación con la acción de los jugos digestivos, como ponemos de manifiesto en la Tabla IV.

Más de un $20 \%$ de alumnos no contestan o dan una respuesta disparatada, como aquellos que indican que los jugos digestivos dan gusto a los alimentos, o los que les atribuyen la función de destruir los gérmenes de la comida. De entre las restantes respuestas destacamos lo siguiente:

Tabla IV

Función de los jugos digestivos en la digestión ( $\%$ ).

\begin{tabular}{|c|c|c|c|c|c|}
\hline Categorías de respuestas & 6? EGE & $8^{\circ} \quad$ E.G8 & 3o BUP & $3^{\circ}$ EUM & Profesores \\
\hline Ryudan a la digestión & 23 & 2.4 & 42 & 8 & 4 \\
\hline Se segregan en la digestión & 1 & 11 & 3 & 8 & $\Delta$ \\
\hline Se mezclan can los alimentos & 17 & 6 & - & 3 & - \\
\hline Cesconpasen los alimentos & 23 & 21 & 30 & 61 & $9 ?$ \\
\hline Otras/NC & 36 & 38 & 25 & 20 & - \\
\hline
\end{tabular}


- En un buen número de casos, el papel de estos jugos es contribuir al proceso digestivo, ayudando a la digestión (ablandando el alimento, facilitando el desplazamiento del bolo alimenticio...), para que la digestión se realice mejor o para facilitarla; o bien, los jugos se mezclan con los alimentos para separarlos o para triturarlos.

- Su participación en la descomposición de los alimentos es reconocida por una proporción relativamente baja de alumnos (notablemente superior en Magisterio). En ocasiones se habla simultaneamente de descomposición o disolución de los alimentos.

En sus respuestas se encuentran, además, bastantes referencias al estómago, haciéndole glándula secretora de los jugos digestivos (uson unos líquidos que se producen en el estómago»), atribuyéndoie un papel central en el proceso digestivo ( $\ldots$... para la descomposición de los alimentos en el estómago»; «... para triturar los alimentos en el estómagon).

Como podemos apreciar en la Tabla V, el jugo pancreático y la bilis son las secreciones más conocidas. Observamos una notable progresión en Magisterio en lo que se refiere a cada secreción individualmente considerada (aproximadamente entre el $70 \%$ y el $80 \%$ de los alumnos recuerdan las secreciones pancreáticas, hepáticas y gástricas). El jugo intestinal resulta ser, en todos los niveles, el menos conocido.

\section{Tabla V}

Conocimiento de los jugos digestivos $\left(\%_{0}\right)$.

\begin{tabular}{|c|c|c|c|c|c|}
\hline jugos digestivos & 59 EGB & 므 ECE & 3? BUP & 32 EUM & Proresores \\
\hline Saliva & la & 13 & 11 & 30 & 31 \\
\hline Jugo gástrico & 53 & 36 & 36 & 67 & 38 \\
\hline Jugo pancreático & 60 & 69 & S6 & 80 & $6 ?$ \\
\hline gilis & 50 & 63 & 53 & 72 & 62 \\
\hline Jugo intestinal & 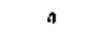 & 22 & - & 30 & 38 \\
\hline Otros/NC & 35 & 38 & 33 & 19 & 46 \\
\hline
\end{tabular}

Resulta sorprendente comprobar el reducido número de alumnos que señalan la saliva como secreción digestiva. Ello se debe a que, aún asociando esta secreción con la alimentación, muchos alumnos opinan que su acción no es otra que la de humedecer el alimento y facilitar el desplazamiento del bolo alimenticio. Esto, en los niveles básicos, es sostenido por la casi totalidad de los alumnos.

Aproximadamente, un $30 \%$ de alumnos de los niveles de EGB y BUP, no responden a esta cuestión, identificándose, en ocasiones, la pepsina, el cuajo $o$ el quimo como secreciones digestivas.

\section{Concepto de absorción}

Finalizamos este trabajo analizando las nociones que poseen los estudiantes sobre el proceso de absorción de los productos resultantes de la digestión. Aunque introducimos una pregunta relativa al destino y utilización de los productos absorbidos, dejamos para un estudio posterior estos aspectos del proceso de nutrición.

Las respuestas al cuestionario, asi como las entrevistas individuales realizadas, nos han permitido aproximarnos al conocimiento de los alumnos sobre 4 aspectos básicos de este proceso:

1. En primer lugar, conocer el órgano en el que piensan que se realiza la absorción de los compuestos resultantes del proceso digestivo. En relación con ello, los resultados que presentamos en la Tabla VI ponen de manifiesto:

a) Aun siendo mayoritarios, en todos los niveles, los alumnos que contestan correctamente, sólo en Magisterio esta cifra es realmente importante.

b) Entre un 10\% y un $15 \%$ opinan que la absorción tiene lugar en el estómago, pensando muchos de ellos, como hemos señalado anteriormente, que la digestión finaliza en el estómago, pasando al intestino los productos de excreción resultantes de la misma («las sustancias malas").

c) Resulta preocupante comprobar cómo en los niveles de EGB y BUP, uno de cada tres alumnos no contestan o dan respuestas atípicas, como las de aquellos

Tabla VI

Lugar donde tiene lugar la absorción $(\%)$.

\begin{tabular}{|c|c|c|c|c|c|}
\hline Parte del tubo digestivo & $\underline{6^{\circ} \mathrm{EGB}}$ & 8들 & $3^{\circ}$ BUP & 3? EUM & Profesores \\
\hline Estómago & 16 & 10 & 11 & 9 & 8 \\
\hline Intestino delgado & 38 & 45 & 39 & 74 & 84 \\
\hline Intestino grueso & 11 & 11 & 3 & $\sigma$ & - \\
\hline otros/NC & 35 & 34 & 47 & $1 !$ & 8 \\
\hline
\end{tabular}


que indican el esófago o la boca (en este último caso, se está confundiendo absorción con ingestión de alimentos).

Con relativa frecuencia los alumnos hacen referencia a las vellosidades intestinales, siendo descritas en EGB como poros, pelillos o glándulas. En Magisterio, por el contrario, casi la mitad de los alumnos realizan una descripción que se podría considerar correcta, mientras que uno de cada cuatro alumnos, sigue manteniendo la idea de que son como pelillos para absorber.

2. El segundo punto de interés está relacionado con la noción que poseen los alumnos del proceso de absorción. Cuando lo explican por escrito aparecen diferentes opciones, tal como reflejamos en la Tabla VII.

a) En muchos casos, con una explicación más o menos precisa, se aprecia en las respuestas de los alumnos la noción de incorporación de sustancias más pequeñas a la sangre, como explicación del proceso de absorción. Esto parece estar claro para un elevado número de alumnos, particularmente de Magisterio, aunque se exprese de manera muy deficiente («asimilación de los alimentos por la sangre»), y se ignore la naturaleza de este proceso («a través de unos poros o pelilloss)).

b) Aproximadamente, un $10 \%$ de los alumnos to describen como el tránsito de alimentos por el tubo digestivo («tomar alimentos que pasan a la digestión», «pa-

Tabla VII

Concepto de absorción (\%)

\begin{tabular}{|c|c|c|c|c|c|}
\hline Cotegorias de respuessas & 6:ECD & $\underline{\theta^{\circ} \mathrm{EGE}}$ & $\underbrace{3^{\circ} \text { BUP }}$ & $\underline{3}$ Eusㅛ & Proresores \\
\hline \multicolumn{6}{|l|}{ Trúnsito de sustancias } \\
\hline a ia sangre, células & $\Delta 2$ & 70 & so & 88 & 96 \\
\hline \multicolumn{6}{|l|}{ Tuma de alimentos; trân- } \\
\hline sito en tubo digestivo & 9 & 11 & 8 & 6 & - \\
\hline \multicolumn{6}{|l|}{ Segaracion de sugtancias } \\
\hline buenes de malas & 12 & - & 3 & - & 4 \\
\hline \multicolumn{6}{|l|}{ Confusión de sossorción } \\
\hline con digestión & 3 & 2 & 3 & - & - \\
\hline $0 t r a s / N C$ & $3 \mathbf{a}$ & 17 & 36 & 6 & - \\
\hline
\end{tabular}

san los alimentos del estómago al intestino delgado»).

c) Por último, observamos cómo un número reducido de alumnos confunden digestión con absorción («descomposición de los alimentos», "separación de las sustancias buenas de las malas»).

Hasta BUP persisten unos niveles relativamente elevados de alumnos que no contestan o que señalan posibilidades bastante insólitas.
3. En la Tabla VIII, presentamos otro aspecto de las respuestas de los alumnos, que aporta datos suplementarios. En efecto, consideramos solamente los alumnos incluidos en la primera de las categorías de la Tabla anterior, es decir aquello que explican el proceso de absorción como la incorporación de compuestos a la sangre, y prestamos atención a las sustancias que, según dichos alumnos, experimentan ese tránsito.

Aunque la mayoria de los alumnos parecen reconocer que lo que se absorbe son las sustancias nutritivas, al menos en los niveles básicos, se desconoce generalmente lo que este término significa con exactitud. Así, se identifican, en ocasiones, con alimentos; o bien, como señalábamos antes, se reconoce que los alimentos contienen únicamente sustancias buenas y malas.

Esto último, unido a la idea bastante generalizada en EGB, de que en la digestión se separan de los alimentos las sustancias buenas («que serán absorbidas»), de

Tabla VIII

Naturaleza de las sustancias absorbidas (\%).

\begin{tabular}{|c|c|c|c|c|c|}
\hline Clase de sustancias & $\underline{\mathrm{G}^{\circ} \mathrm{ECO}}$ & $\theta^{\circ} E G S$ & 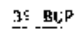 & 39 Elik & Profesoress \\
\hline Sustancuas nutritivas & 55 & SI & 61 & so & 96 \\
\hline Sustanc:as buenas & 9 & 13 & 5 & 7 & .. \\
\hline Sustancias necesarias & - & 7 & 16 & 7 & a \\
\hline Alimentos turenos, nJ- & & & & & \\
\hline tritivos & 30 & $p_{\mathbf{i}}$ & $3 !$ & 3 & - \\
\hline Productos & 6 & - & 6 & 3 & .. \\
\hline Jusos de los alisentos, & - & 8 & 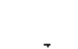 & - & - \\
\hline
\end{tabular}

las malas («que van al intestino»), resalta la idea de que «nunca se absorben Ias sustancias perjudiciales».

P. ¿Pueden ser absorbidas las sustancias malas o perjudiciales?

Cuatro alumnos: ¡No!

$P$. Entonces, una sustancia venenosa o que pudiera causar daño al organismo no sería absorbida.

J.M. No se absorbe, o no se llega a absorber toda... pero llega al corazón por la sangre.

G.C. Bueno, sí..., pero tiene una acción tan rápida que te mueres antes de que salga del estómago.

A.P. Se puede absorber por equivocación.

S.M. El veneno sí se absorbe porque los jugos gástricos no lo pueden destruir.

$\mathrm{P}=$ profesor; JM, GC, AP y $\mathrm{SM}=$ alumnos de octavo de EGB. 
4. La mayor parte de los alumnos explican, con mayor o menor precisión según el grado de instrucción, que las sustancias absorbidas son transportadas por medio de la sangre a las células (al cuerpo), para la obtención de energía, para la nutrición de las células o para el metabolismo. No obstante, en EGB existen dificultades para responder por escrito a estas cuestiones.

\section{Anthisis compurativo de los distintos niveles estudiados}

No er el proposito fundamental de nuestro trabajo la realización de un estudio comparativo, entre los diferentes niveles educativos, sobre el grado de aprendizaje de los conceptos implicados en estas lecciones. Sin embargo, los resultados obtenidos nos permiten extraer algunas conclusiones, de entre las que resaltamos las siguientes:

a) Aunque los alumnos incluidos en nuestro estudio representan un amplio rango de edades (entre los 11 y los 21 años), con niveles de formación notablemente distintos, observamos con ligeras variaciones, 12 persistencia de un modelo uniforme para todos ellos, cunndo analizamos los valores medios relativos 2 la importancia de las acciones en los diferentes or ganos del tubo digestivo. En este modelo, el estómigo se sitúa invariablemene como el más destacado en la digestión. Como cambios más notables aì comparar niveles (figura 1), podemos destacar el incremento en la impor-

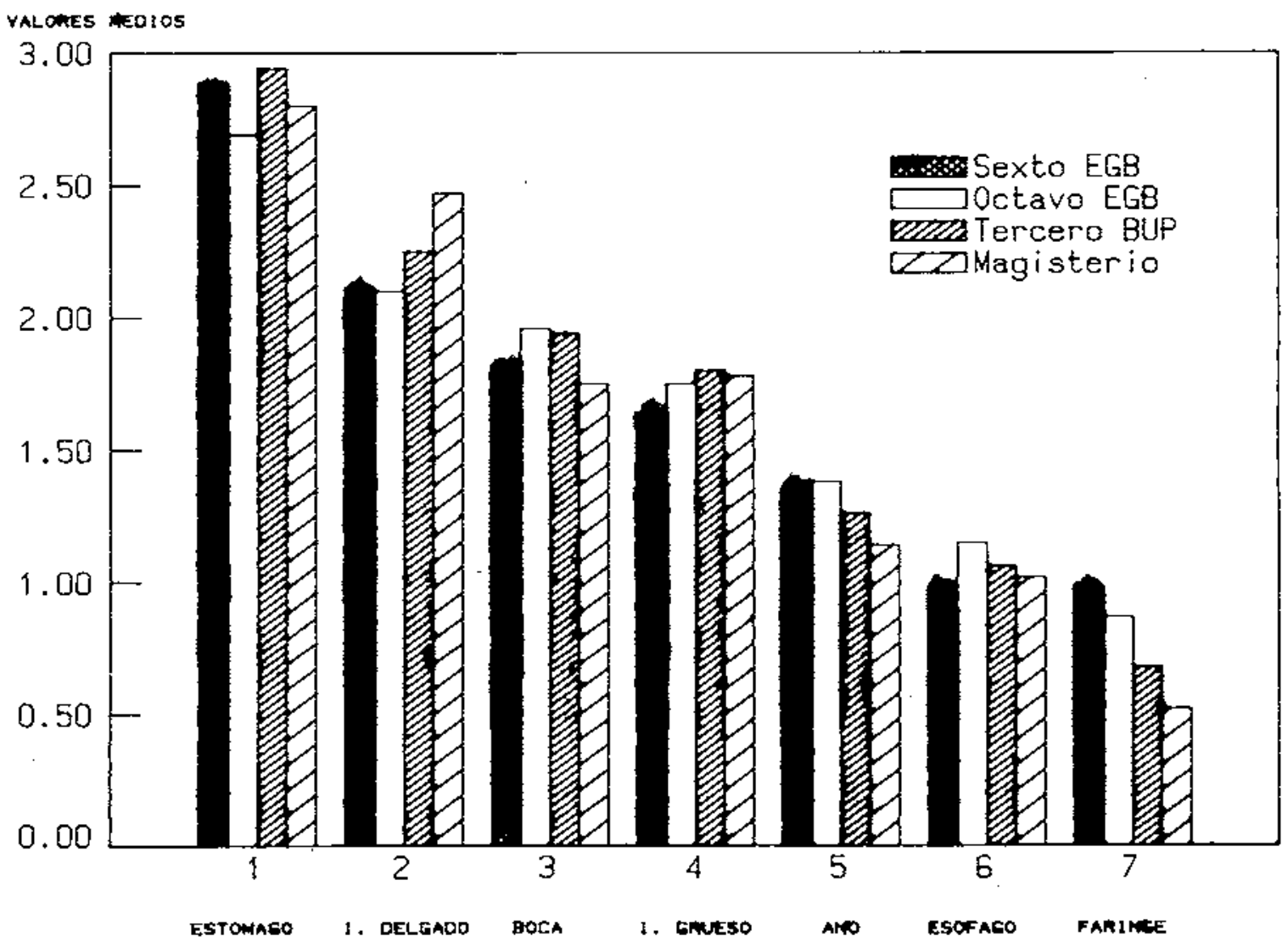


tancia que se concede al intestino delgado y el descenso en la consideración del papel atribuido a la faringe.

Al orientar nuestro trabajo en el sentido de establecer comparaciones entre distintos órganos, particularmente entre el estómago y el intestino delgado, sólo intentamos poner de manifiesto las ideas de los alumnos al respecto, consecuencia en buena medida de experiencias extraescolares, aún siendo conscientes de que estas comparaciones pueden no resultar válidas al establecer planteamientos educativos determinados.

b) En relación con el concepto de digestión y el papel que corresponde a los jugos digestivos en este proceso, observamos una progresión importante, a medida que aumenta el nivel de instrucción. En este sentido, aunque resultan pequeñas las diferencias que existen cuando comparamos el número que señala como correcta la frase «descomponer los alimentos en sustancias nutritivas», mediante sus explicaciones escritas o verbales podemos comprobar cómo en los niveles básicos no está suficientemente diferenciado el concepto de alimento, en relación con el de sustancia nutritiva (son las "sustancias buenas»), ni se entiende que dicha descomposición suponga algo más que una separación, por procedimientos fisicos que dan como resultado una disminución del tamaño de las particulas que forman parte del alimento, de las sustancias buenas y malas que éste contiene. En Magisterio, sin embargo, esta opinión va siendo sustituida por otra, que considera este proceso como un conjunto de acciones físicas y químicas, que producen como resultado la descomposición del alimento en sustancias más sencillas. Sin embargo, enttre estos alumnos universitarios persisten algunos errores e imprecisiones sobre otros aspectos importantes, que cuestionan que el aprendizaje sobre los mismos haya tenido la suficiente significación, a pesar de haber estudiado estos procesos en reiteradas ocasiones.

c) En relación con la absorción de los productos resultantes de la digestión, se aprecia también una progresión notable, tanto en lo que se refiere a la localización del proceso como a su naturaleza («incorporación de sustancias nutritivas a la sangre»).

Por último, hay que señalar que los resultados obtenidos a partir de una reducida muestra de profesores (maestros, profesores de enseñanzas medias y universitarias, todos del área de ciencias), muestran cómo se han corregido, casi en la totalidad de los casos, las nociones erróneas que sobre estos procesos mantienen los alumnos de los niveles básicos, al menos cuando nos referimos a ellos en sus aspectos más generales, como es el caso que nos ocupa.

\section{Algunas consideraciones educativas}

Como señalábamos antes, establecidos los conocimientos previos de los alumnos, y después de comprobar su persistencia pese a los métodos habituales de enseñanza (debido en buena medida a la coherencia que en ocasiones les caracteriza), es necesario un replanteamiento fundamentado de la enseñanza de las Ciencias (Gil 1987).

En nuestra opinión, dicho replanteamiento deberá tener en cuenta, al menos, algunos puntos de referencia que parecen importantes:

a) En primer lugar, lo que el alumno es capaz de aprender. ¿Pueden los alumnos de Ciclo Superior de EGB alcanzar nociones significativas en relación con los procesos de digestión y absorción? Y, en caso afirmativo, ¿qué planteamientos didácticos podrían ser apropiados para ello?

Sin estar de acuerdo en todos los supuestos con quienes opinan que introduciendo ciertos conceptos en edades tempranas se facilita el aprendizaje posterior de los mismos (esa pretendida facilidad puede convertirse rápidamente en obstáculo que dificulta aprendizajes posteriores), es preciso reconocer la importancia que los programas oficiales conceden al conocimiento del cuerpo humano, núcleo fundamental en la enseñanza de las Ciencias en la educación básica.

b) El interés didáctico de los errores de los alumnos. Como señala Giordan (1985), se aprende no sólo «contra», sino también "con» y «gracias a» los errores.

c) Determinados supuestos básicos en relación con los mecanismos de aprendizaje de los conceptos cientificos por parte de los alumnos (Posner y col. 1982, Novak 1982, Driver 1986). Así, en opinión de Posner y sus colaboradores, el cambio conceptual se facilita cuando surgen contradicciones entre las nociones de los alumnos y los resultados de las experiencias de aprendizaje, siendo así que otras explicaciones nuevas, más inteligentes, plausibles $y / o$ fructiferas, sustituyen a las anteriores.

En el tema que nos ocupa, y en oposición a una ensenanza excesivamente compartimentada, donde los conceptos o procesos se presentan escasamente relacionados entre sí, es necesario realizar planteamientos más amplios para grupos de lecciones. Es frecuente comprobar cómo muchos libros de texto y bastantes profesores abordan el estudio de los procesos implicados en la nutrición humana, centrando más su atención en el conocimiento de cada uno de los aparatos o sistemas que intervienen (digestivo, circulatorio...), que sobre las relaciones que existen entre ellos.

En nuestra opinión, los mapas de conceptos (Novak 1984) pueden resultar instrumentos de trabajo de gran utilidad para destacar aquellos conceptos o procesos más relevantes en la nutrición y establecer entre ellos relaciones suficientemente significativas. No obstante, de acuerdo con Arnaudin y Mintzes (1985), pensamos que es necesario continuar con los trabajos que pongan de manifiesto las concepciones alternativas de los estudiantes, y en nuestro caso particular sobre el conjunto de lecciones que componen el bloque temático 
de la nutrición humana, para propiciar un aprendizaje significativo de los conceptos científicos.

No queremos finalizar este trabajo sin exponer alguna de las reflexiones que nos han ido surgiendo durante su desarrollo, y que, de alguna manera, podrían ser de utilidad para el profesor cuando aborda el estudio de la digestión con los alumnos de niveles básicos:

a) Los errores de los alumnos en relación con la anatomía del aparato digestivo, puestos de manifiesto en un trabajo anterior (Banet y Nuñez 1988), dificultan el aprendizaje de estos procesos. Así, por ejemplo, situar de forma correcta el lugar del tubo donde segregan el higado y el páncreas, asi como localizar el lugar en que se produce la absorción de las sustancias, constituyen referencias importantes para propiciar el cambio en aquellos alumnos que poseen la noción de que el estómago es el órgano central (en ocasiones el único) del proceso digestivo.

b) $\mathrm{Si}$ ciertas lecciones de anatomía humana pueden

\section{REFERENCIAS BIBLIOGRÁFICAS}

ARNAUDIN, M.W. y MINTZES, J.J., 1985. Students alternative conceptions of the human circulatory system: A cross-age study, Sciencie Education, Vol, 69 (5), pp. $721-733$.

BANET, E. y NÚN̄EZ, F., 1988. Ideas de los alumnos sobre la digestión: I. Aspectos anatómicos, Enseñanza de las Ciencias, Vol. 6 (1), pp. 30-37.

COLL, C., 1986. Un marco de referencia para el currículo escolar, Cuadernos de Pedagogía, Vol. 139, pp. 12-16.

DRIVER, R., 1986. Psicología cognoscitiva y esquemas conceptuales de los alumnos, Enseñanza de las Ciencias, Vol. $4(1)$, pp. 3-15.

GIL, D., 1986. La metodologia cientifica y la enseñanza đe las ciencias. Unas relaciones controvertidas, Enseñanza de las Ciencias, Vol. 4 (2), pp. 111-121. ofrecer serias dificultades para los alumnos de enseñanza básica, particularmente cuando existe imposibilidad de ponerlos en relación directa con el objeto de estudio, éstas se incrementan cuando nos referimos a procesos basados en esos conocimientos anatómicos. En relación con ello, estamos analizando la eficacia para el aprendizaje del proceso digestivo y adaptándolas, en todo caso, al nivel de los alumnos.

c) La diferenciación entre alimento y sustancia nutritiva es importante para alcanzar un conocimiento adecuado del proceso digestivo y de la absorción.

d) Dependiendo del nivel de instrucción de los alumnos, será necesario insistir sobre la naturaleza de los productos resultantes de la digestión, su incorporación a la sangre y su destino posterior (más del $90 \%$ de los alumnos de Magisterio desconocen que el hígado es el receptor inmediato de gran número de las sustancias absorbidas, ignorando las complejas funciones que esta glándula desarrolla).
GIL, D., 1987. Los errores conceptuales como origen de un nuevo modelo didáctico: de la búsqueda a la investigacion, Investigación en la Escuela, 1, pp. 35-41.

GIORDAN, A., 1982. La enseñanza de las ciencias. (Pablo del Rio: Madrid).

GIORDAN, A., 1985. Interés didáctico de los errores de los alumnos, Enseñanza de las Ciencias, Vol. 3 (1), pp. 11-17.

NOVAK, J.D., 1982. Teoría y práctica de la Educación. (Alianza Editorial: Madrid).

NOVAK, J.D. y GOWIN, D.B., 1984. Learning how to learn. (Cambridge University Press: Cambridge).

POSNER, G.J., STRIKE, K.A., HEWSON, P.W. y GERTZOG, W.H., 1982. Accomodation of a scientific conception: Toward a theory of conceptual change, Sciencie Education, Vol. 66, pp. 211-228. 